\title{
Pacinian representations of fine surface texture
}

\author{
SLIMAN BENSMAÏA and MARK HOLLINS \\ University of North Carolina, Chapel Hill, North Carolina
}

\begin{abstract}
Subjects were presented with pairs of finely textured stimuli and were instructed to rate their dissimilarity, using free magnitude estimation. The subjects also rated the stimuli along each of four textural continua: roughness, hardness, stickiness, and warmth. In subsequent experimental sessions, we used a Hall effect transducer to measure the vibrations produced in the subjects' fingertip skin as the stimuli were scanned across it. We wished to assess the extent to which the perceptual dissimilarity of the textures could be explained in terms of the perceptual dissimilarity of the vibrations they elicited in the skin. To that end, we invoked a model characterizing the Pacinian representation of a vibratory stimulus. From the model, we computed the difference in the vibratory representations of the two stimuli in each pair. We found that the bulk of the variance in perceived dissimilarity of the textures was accounted for by differences in the Pacinian representations of the vibrations they produced. Our results further suggested that the textural information conveyed by the Pacinian system concerns surface roughness and, possibly, stickiness.
\end{abstract}

It is not uncommon in everyday experience to identify a material on the basis of its texture. We can readily recognize a satin sheet or a page of cotton paper by running a finger across it. Textures range from relatively coarse (such as those of Braille patterns or low-grit sandpapers) to extremely fine (such as those of frosted glass or silk). Researchers have sought to understand the chain of events leading from stimulus to textural percept. Katz (1925/1989) proposed that two distinct mechanisms mediate the perception of coarse and fine textural features. On the one hand, he argued, the configuration and relative prominence of coarse textural elements, such as the veins on the back of a leaf, are spatially encoded. On the other hand, fine textural elements, such as the fibers in writing paper, are perceived on the basis of the small vibrations they induce in the exploring skin, which stimulate what he called "the vibration sense."

\section{A Spatial Code for Coarse Textures}

The notion that coarse textures are encoded in terms of the spatial layout of their elements has received strong experimental support in a variety of contexts. Most of this work has focused on roughness, because of the salience of this perceptual dimension. In the first modern study of roughness coding, Lederman and Taylor (1972) had subjects estimate the roughness of tactile gratings with grooves and ridges that varied in width from 0.125 to $1.0 \mathrm{~mm}$. Lederman and Taylor found that roughness increased monotonically with groove width but decreased as ridge

This work was supported by the Cognitive Science Program of the University of North Carolina at Chapel Hill. We thank Marcus Johnson for his assistance in developing the methodology and Kerry Ledoux for careful reading of the manuscript. Correspondence concerning this article should be addressed to S. Bensmaïa, Mind/Brain Institute, Johns Hopkins University, 3400 N. Charles St., 338 Krieger Hall, Baltimore, MD 21218 (e-mail: sliman@mail.mb.jhu.edu). width increased. Subsequently, Taylor and Lederman (1975) developed a model of an idealized finger pressed onto a grooved surface and derived a set of skin deformation parameters that might underlie the perception of roughness. They found that the instantaneous pattern of indentation of the skin produced by a given texture determines its roughness. Specifically, they proposed that perceived roughness is a function of the volumetric displacement of skin from its resting configuration while touching a given surface. Significantly, their model postulated that these representations rely on essentially static patterns of deformation. The speed with which the texture is scanned is thus relevant to roughness perception only to the extent that it affects the instantaneous pattern of skin indentation.

In an attempt to explicitly link the textural percept to patterns of activation in populations of mechanoreceptive afferents, Connor, Hsiao, Phillips, and Johnson (1990) recorded the activity in monkey slowly adapting type 1 (SA1), rapidly adapting (RA), and Pacinian (PC) afferents evoked by plastic surfaces embossed with dot arrays of varying dot diameter and center-to-center spacing. They also had human subjects provide magnitude estimates of the roughness of these same surfaces. Their methodology consisted of seeking aspects of the peripheral representation (in monkeys) that were associated with roughness perception (in humans). Their physiological method allowed them to reconstruct the spatiotemporal pattern of activity evoked in each class of primary afferents by the embossed surfaces. Assuming that the peripheral representations in monkey approximate those in humans, they assessed which of four classes of neural codes could account for their psychophysical data.

The candidate neural codes that most closely matched roughness estimates as a function of dot diameter and interdot spacing were temporal and spatial variation in afferent firing rates, indicating that it is not overall neural 
activity that determines perceived roughness but, rather, the degree to which this activity varies over time or space.

Connor and Johnson (1992) conducted another study to distinguish between temporal and spatial hypotheses. They chose their stimulus set in such a way that the two hypotheses made contrasting predictions as to the roughness of the surfaces. In one condition, the distance between dots varied only along the dimension in which the surfaces were scanned (horizontal condition); in the other condition, the distance between dots varied along the dimension perpendicular to the scanning direction (vertical condition). The temporal variation hypothesis predicted that roughness would increase as the distance between the dots increased in the horizontal condition but would decrease as interdot distance increased in the vertical condition, because fewer afferents would be stimulated. The spatial variation hypothesis predicted that roughness would increase with interdot distance in both the horizontal and the vertical conditions. Connor and Johnson found that roughness increased in both the horizontal and the vertical conditions and concluded that spatial variation determined perceived roughness. These results were ambiguous, however, as to the underlying receptor systems, in that the pattern of spatial variation in both SA1 and RA firing rates could account for the roughness estimates.

To determine which of these two receptor types mediates roughness perception, Blake, Hsiao, and Johnson (1997) varied the height and width of the embossed dots. They found that, in the case of small diameter dots, height had little effect on RA firing rates but had a substantial effect on both roughness estimates and SA1 firing rates. They concluded that roughness perception is mediated by SA1 afferents.

Yoshioka, Gibb, Dorsch, Hsiao, and Johnson (2001) tested the applicability of the spatial hypothesis to the perception of finer surfaces by again combining psychophysical and neurophysiological methods, this time to study tactile gratings with spatial periods as small as $0.1 \mathrm{~mm}$. They found that spatial variation in SA1 firing rates could account for ratings of perceived roughness for even their finest surfaces and concluded, invoking the principle of parsimony, that only one mechanism underlies roughness perception.

Lederman, Loomis, and Williams (1982) also cast doubt on the contribution of the vibrotactile system to the perception of texture, in a study in which the effects of vibrotactile adaptation on perceived roughness were investigated. They had subjects rate the roughness of tactile gratings with spatial periods (i.e., 1 groove +1 ridge) ranging from 0.6 to $3 \mathrm{~mm}$. In the experimental condition, the subject's exploring finger was subjected to sustained and intense vibratory stimulation before the psychophysical task. The stimulus frequency was manipulated so that either the RA or the PC channel became selectively adapted. They found that vibrotactile adaptation reduced the perceived intensity of supraliminal vibratory stimuli, indicating that their manipulation was successful. In contrast, the perceived roughness of the gratings was relatively unaffected when either channel was adapted. Lederman et al. concluded that the perception of roughness does not rely on vibrotaction.

This formidable body of evidence indicated, then, that under all studied conditions, roughness perception (1) relies on a spatial code and (2) is mediated fairly exclusively by the SA system. However, in the aforementioned research, the textured stimuli comprised relatively coarse elements: Embossed dots ranged in height from 0.28 to $0.62 \mathrm{~mm}$ and in diameter from 0.25 to $2.5 \mathrm{~mm}$ across studies (Blake et al., 1997; Connor et al., 1990; Connor \& Johnson, 1992), and gratings ranged in spatial period from 0.10 to $3.0 \mathrm{~mm}$ (Lederman et al., 1982; Lederman \& Taylor, 1972; Taylor \& Lederman, 1975; Yoshioka et al., 2001). But many common surfaces consist of textural features that are orders of magnitude smaller than these.

\section{Vibrotactile Coding of Fine Textures}

When texture perception was studied with finer surfaces, evidence of vibrotactile coding emerged, which led Hollins, Bensmaïa, and Risner (1998) to revive Katz's (1925/1989) idea that texture perception relies on two distinct mechanisms. According to this updated formulation, called the duplex theory of texture perception, the perception of coarse textural features is mediated by the SA system (as evidenced by the research presented above), whereas that of textures with spatial periods below about 200 microns relies on vibrotaction (specifically, on the PC channel).

Several lines of evidence implicate vibrotaction in fine-texture perception. Hollins and Risner (2000) had subjects discriminate two fine (particle size $<20 \mu \mathrm{m})$ and two coarse (particle size $>100 \mu \mathrm{m}$ ) abrasive papers in two conditions. In the movement condition, the surface was scanned across the subject's finger; in the stationary condition, the subject was instructed to press his or her finger against a motionless surface. For the coarse surfaces, discrimination was found to be equivalent in the movement and stationary conditions, but fine surfaces were discriminable only in the movement condition. Hollins and Risner attributed the poor performance for fine-texture discrimination in the stationary condition to the fact that no vibrations were produced because the finger was motionless with respect to the surface. Since the perception of fine textures relies on these vibrations, the textural percept was severely degraded in their absence. In contrast, because the perception of coarse textures does not rely on vibrations but, rather, relies on static patterns of skin deformation, the ability to discriminate these was not affected by the lack of movement.

Hollins and Risner (2000) next asked the subjects to estimate the roughness of abrasive papers ranging in particle size from 9 to $350 \mu \mathrm{m}$, presented under both movement and stationary conditions. They found that there was a threefold difference in roughness between the 9- 
and the $100-\mu \mathrm{m}$ sandpapers in the movement condition, but almost no difference in the stationary condition. In contrast, the slope of the psychophysical function for the coarser surfaces (i.e., for those with particles $>100 \mu \mathrm{m}$ in size) did not differ between the movement and the stationary conditions.

Attacking the problem from another angle, Hollins, Fox, and Bishop (2000) investigated the perceptual consequences of introducing, rather than eliminating, vibrations. They presented subjects with two identical grooved surfaces with spatial period of $298 \mu \mathrm{m}$. On certain trials, one of the two surfaces was vibrating. The subjects were asked to judge which of the two surfaces felt smoother. The investigators found that the vibrating surface tended to be perceived as rougher than the (otherwise identical) static surface and that this tendency increased as the vibrations became more intense. These findings demonstrate that vibrations can influence perceived texture, as is predicted by the duplex theory.

Hollins, Bensmaïa, and Washburn (2001) investigated more explicitly the peripheral mechanisms underlying fine-texture perception. They had subjects discriminate etched silicon wafers that differed in spatial period. In the first experiment, the spatial period of the standard was $40 \mu \mathrm{m}$, and that of the comparisons varied from 16 to $80 \mu \mathrm{m}$, all stimuli thus falling within the range of fine textures. In the experimental condition, the subjects were presented with a $100-\mathrm{Hz}$ vibratory stimulus designed to adapt both RA and PC channels prior to the discrimination task. No adaptor was presented in the control condition. The subjects were able to discriminate the surfaces in the unadapted condition but were at chance in the adapted condition. When the same experiment was conducted using coarse surfaces (a standard of 2,172 $\mu \mathrm{m}$ and comparisons ranging from 944 to $3,200 \mu \mathrm{m}$ ), discrimination was not affected by adaptation. These results provided strong evidence that the discrimination of fine textures is mediated by the vibrotactile system, whereas that of coarse textures is not.

Hollins et al. (2001) proceeded to elucidate which of the two main vibrotactile channels - RA or PC - was implicated in fine-texture discrimination by adapting the channels individually, with high-intensity vibratory stimuli of 10 and $250 \mathrm{~Hz}$, respectively. Hollins et al. (2001) found that adaptation at $10 \mathrm{~Hz}$ did not substantially impair discrimination (as compared with a control condition), whereas adaptation at $250 \mathrm{~Hz}$ abolished it. They concluded that the perception and discrimination of the fine surfaces was mediated by the PC channel.

Finally, Bensmaïa and Hollins (2003) recorded the vibrations elicited in the skin as etched silicon wafers with spatial periods ranging from 16 to $80 \mu \mathrm{m}$ were scanned across subjects' fingerpads. In separate sessions, the subjects judged the roughness of these same surfaces. Predictably - and therefore confirming the validity of these novel recordings - the frequency of the dominant spectral component of the vibrations elicited by a surface was found to be inversely related to the surface's spatial period (with the speed of movement held constant); in contrast, the intensity of the vibrations were found to increase with spatial period. (Following Makous, Friedman, \& Vierck, 1995, we used, as a measure of intensity, the overall Pacinian-weighted power of a vibration.) Furthermore, roughness was found to be proportional to the intensity of the textural vibrations. Since our results showed that both the intensity and the temporal frequency of the vibration produced by a surface are systematic functions of its spatial period, both temporal and intensive codes were viable candidates to underlie roughness, perception. In a subsequent experiment, Bensmaïa and Hollins (2003) varied the scanning velocity in order to differentially manipulate the temporal and intensive characteristics of the texture-induced vibrations. We found that doubling the scanning velocity did not produce a consistent decrease in perceived roughness, as predicted by the temporal hypothesis. On the other hand, the complex effects of speed on roughness matched those of speed on power. We concluded that the roughness of a fine surface is a function of the intensity of the vibrations it elicits in the skin. Given that the intensity measure was scaled according to the spectral sensitivity of the Pacinian system, the close match between intensity and roughness further supported the hypothesis that the Pacinian system mediates the perception of fine texture.

\section{Rationale for the Present Study}

The objective of the present study, then, was to more fully characterize the ways in which the PC system conveys information about the properties of a touched surface. This channel's involvement in roughness perception has already been described; however, surfaces vary along other sensory continua as well, and the perception of these may or may not rely on vibratory signals. For instance, soft/hard appears to be a universal perceptual dimension, and sticky/slippery and warm/cool are salient dimensions for some individuals (Hollins, Bensmaïa, Karlof, \& Young, 2000; Hollins, Fox, \& Bishop, 2000).

Our approach consisted in measuring the perceived dissimilarity of textured surfaces and assessing to what extent it could be accounted for by differences in the Pacinian representations of the vibrations produced by movement of these surfaces along the finger. We considered whether the vibrotactile contribution to the percept was determined solely by intensive properties of the vibrations, or whether temporal factors also played a role in shaping it. We also assessed whether differences in surface stickiness, hardness, roughness, and warmthmeasured psychophysically - could be accounted for by differences in the spectral properties of the movementinduced vibrations. Surface warmth was measured primarily in order to determine the extent to which this 
property contributed to the overall percept and as a control for our methodology, since a vibratory code for thermal conductivity is highly unlikely.

\section{METHOD}

\section{Subjects}

The 5 subjects were graduate or professional students ( 2 females and 3 males) from 24 to 31 years of age. Four of the subjects were paid for their participation. The 5 th subject was the first author. ${ }^{1}$

\section{Stimuli}

The stimuli were 12 finely textured surfaces, 7 of which were composed of everyday materials, such as paper, cloth, and wood. The rest were manufactured abrasives (3M, St. Paul, MN) and etched silicon wafers of the kind used in previous studies (Bensmaïa \& Hollins, 2003; Hollins et al., 2001). The stimuli are listed in Table 1. Surfaces were chosen to be smooth enough to (presumably) fall within the Pacinian-mediated range, while having textural features large enough to produce measurable vibrations in the skin. ${ }^{2}$ Data obtained from one surface - namely, the back of the lapping film - were discarded when it was discovered that this surface had been damaged in the course of the psychophysical experiments. Subsets of textures, such as silk charmeuse and satin, or cedar, birch, and paper, were chosen to be similar to each other but substantially different from other surfaces, to ensure that the stimulus set would yield a wide range of dissimilarities, both psychophysically and spectrally.

\section{Apparatus}

The apparatus - similar to that described in Bensmaïa and Hollins (2003) - consisted of a flat belt (HAM-5P, Page Belting Company, Concord, $\mathrm{NH}$ ) on pulleys, driven by a gear motor (Bodine Electric Co., Chicago, IL), which scanned textured surfaces across the subject's stationary finger. The speed of the belt was proportional to a DC voltage produced by a digital-to-analog converter (CIODAS1602/16, ComputerBoards, Mansfield, MA), under the control of a computer (Dell OptiPlex GX1P, Round Rock, TX). Motor speed was measured via an encoder (RM15, RENCO Encoders, Goleta, CA), the output of which was sampled at $50 \mathrm{KHz}$ by a 16-channel analog-to-digital converter (ADC; CIO-DAS1602/16, ComputerBoards). Belt speed was calibrated prior to each psychophysical and recording block.

Texture-elicited vibrations were measured by means of a Hall effect transducer (HET; Type SS94A1F, Honeywell, Morristown, $\mathrm{NJ}$ ), which produces a small current proportional to the distance between it and a magnet. A small circular rare earth magnet (diameter $=5 \mathrm{~mm}$, thickness $=1.6 \mathrm{~mm}$ ) was secured with rubber cement to the center of the distal index fingerpad; the HET was mounted

Table 1

List of the Textured Surfaces

\section{China silk}

Etched silicon—spatial period $=80 \mu \mathrm{m}$

Etched silicon-spatial period $=124 \mu \mathrm{m}$

3M lapping film, back surface

$3 \mathrm{M}$ lapping film - particle size $=30 \mu \mathrm{m}$

$3 \mathrm{M}$ lapping film - particle size $=12 \mu \mathrm{m}$

Latex (from exam gloves)

Cotton paper

Satin

Silk charmeuse

Wood (birch)

Wood (poplar) opposite the magnet, on a metal bar that ran parallel to the palmar surface of the index finger, but was separated from it by about $8 \mathrm{~mm}$ (see Figure 1.) This bar was held in place by a Delrin ring (placed around the middle phalanx and extending to the distal interphalangeal joint), through which it passed. A second metal bar (sans HET) passed along the dorsal surface of the finger to stabilize it. This device fixed the distance between the magnet and the HET at approximately $6 \mathrm{~mm}$, despite involuntary movements of the finger, and thus substantially decreased run-to-run variability in the recording gain, as compared with that used in Bensmaïa and Hollins (2003).

Vibrations produced in the fingertip skin, as textured surfaces were drawn across it, thus traveled up the finger a short distance before causing the magnet to vibrate. The signal current produced in the HET by movements of the magnet was amplified (CP302, Sable Systems, Las Vegas, NV), sampled at $50 \mathrm{KHz}$ by the ADC, and stored to file. For a description of the HET response characteristics, see Bensmaïa and Hollins (2003).

The textured surfaces were mounted on $7.5 \times 7.5 \mathrm{~cm}$ Plexiglas blocks. The height of the blocks varied depending on the thickness of the textured surface, so that the height of each surface-block assembly was $2 \mathrm{~cm}$. The mounted surfaces were fixed, up to three at a time, on a $47 \times 10 \times 2.5 \mathrm{~cm}$ Plexiglas block in which three $7.5 \times$ $7.5 \times 2 \mathrm{~cm}$ square wells had been milled. The surface of the platform was approximately flush with that of the stimuli. If fewer than three surfaces were presented in a given sweep, any empty well was plugged using $7.5 \times 7.5 \times 2 \mathrm{~cm}$ Plexiglas blocks.

In addition, there was a small circular well, $2.5 \mathrm{~cm}$ in diameter and $1.25 \mathrm{~cm}$ deep, in the section of the stimulus assembly preceding the first large well. A load transducer (Model 13/2446-06, Sensotec) was fixed to the center of this small well, and a $2.5-\mathrm{cm}-$ diameter Plexiglas lid was placed over it (thickness $=3 \mathrm{~mm}$ ). The surface of the platform was flush with the top of the lid. Any pressure exerted on the circular lid produced a current in the load transducer, which could then be sampled using an ADC (CIO-DAS1602/16, ComputerBoards) and converted to grams. The load transducer was calibrated before every psychophysical or recording block.

\section{Psychophysical Procedures}

Dissimilarity rating. Before each run, the subject was blindfolded, to eliminate visual cues, and pink noise was presented through earphones to mask laboratory sounds, especially faint sounds that might be produced by movement of the surfaces across the finger. The subject placed his or her arm on an armrest spanning the belt, allowing the index finger to rest lightly on the stimulus assembly near its leading edge. He or she was instructed to maintain this pressure within and across trials.

During a trial, the belt moved past the finger at approximately $3.3 \mathrm{~cm} / \mathrm{sec}$. The force exerted by the subject on the platform was measured as his or her finger scanned over the load transducer. The subject was instructed to rate the dissimilarity of the two surfacespositioned in the first and second wells of the stimulus assemblyon a ratio scale, with 0 signifying that the two surfaces felt identical. Ratings were provided orally to the experimenter. The pairs of surfaces were presented in pseudorandom order, and each surface was presented an equal number of times in the first and the second intervals. In each of six runs, all possible pairs were presented. Ratings obtained in the first run were not included in the analysis; thus, the dissimilarity data consisted of five ratings per pair per subject.

Adjective ratings. Wearing blindfolds and earphones, the subjects were presented with one surface at a time by means of the belt-pulley assembly. After each stimulus presentation, they verbally provided the experimenter with a rating of the texture, along one of four continua (see Hollins, Bensmaïa, et al., 2000): smoothness/ roughness, softness/hardness, slipperiness/stickiness, and coolness/ warmth. The subjects were to assign a higher numerical rating to a texture as its roughness, hardness, stickiness, or warmth increased. 


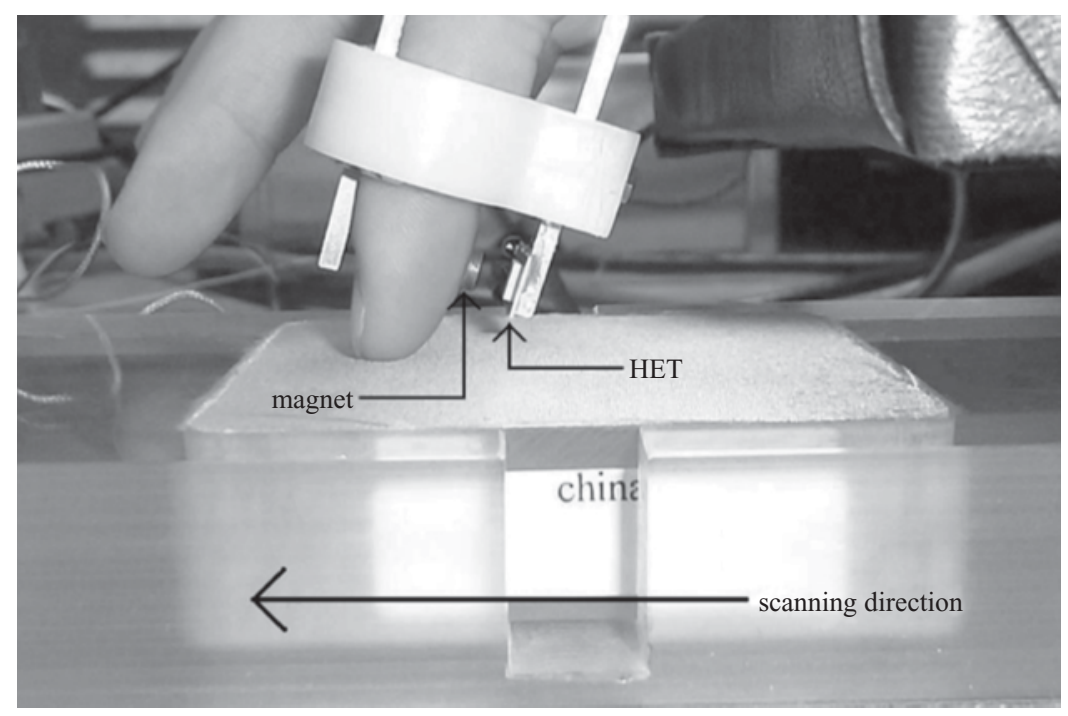

Figure 1. Experimental set-up. The Hall effect transducer (HET) was fixed to a metal bar extending distally from the Delrin ring. The distance between the magnet and the HET was thus held constant during a recording block. The metal bar on the dorsal surface helped stabilize the finger.

Roughness and stickiness were rated on ratio scales; warmth and hardness on interval scales. For the latter two scales, the subjects rated the stimuli along a continuum ranging from cool to warm or from soft to hard. The subjects rated all 12 surfaces along a given continuum before switching to another continuum. Surfaces were presented in pseudorandom order, and the order of the continua along which the subjects rated the surfaces was chosen randomly. In each of six runs, one rating was obtained along each continuum for each surface. Ratings obtained in the first run were not included in the analysis; thus, the adjectival data consisted of five ratings per stimulus per continuum per subject.

All psychophysical measurements were obtained from a given subject before beginning the recording phase.

\section{Recording Procedure}

The subject-his or her arm on the armrest-rested the index finger lightly on the platform. He or she was instructed to maintain a constant pressure within and across recording sweeps. The recording trial began when the belt began to move. The speed of the belt was measured three times during each trial, once before each sampling interval. If any speed measurement fell outside of $15 \%$ of the nominal speed $(3.3 \mathrm{~cm} / \mathrm{sec})$, the recordings were discarded and replaced. When the subject's finger scanned across the load transducer assembly, the force exerted by the subject on the platform was measured. If the force differed by more than $33 \%$ from the mean force exerted by that subject during the psychophysical experiments, the recordings were discarded and replaced. The mean forces (standard deviations) applied by the 5 subjects were 8.6 (4.2), 9.1 (4.0), 13.5 (4.9), 33.1 (9.5), and 25.8 (7.0) g.

As the finger scanned across each of the three surfaces, the amplified signal from the HET was sampled at $50 \mathrm{KHz}$ for $500 \mathrm{msec}$ by means of the ADC and was stored to file. The recording procedure was designed to parallel the psychophysical procedure, except for (1) the presence of the ring and magnet and (2) the number of surfaces presented on a single trial: Two surfaces were presented in each dissimilarity scaling sweep, and one surface was presented in each adjective rating sweep, whereas three were presented in each recording sweep.
Each recording trial consisted of 10 sweeps of the three surfaces, in a fixed order. The middle surface was always the null surfacea completely smooth silicon surface (see Bensmaïa \& Hollins, 2003) - from which a baseline measure of mechanical and electrical noise was obtained for that sweep. A recording block consisted of six recording trials, in each of which a different pair of the textured surfaces was presented. There were five recording blocks per subject. The order of presentation of surfaces was counterbalanced as nearly as possible.

The recording procedure yielded, for each subject, 50 recordings from each textured surface, with a recording obtained from the null surface on each sweep. The top panel of Figure 2 shows individual records obtained from four surfaces, one of which was the null surface. The bottom panel shows segments of the same records on an expanded time scale, in order to highlight differences in waveform.

\section{Analysis}

Because both psychophysical ratings and vibration recordings were similar across subjects, data analyses were carried out on pooled data. We also carried out analyses using individual data and obtained equivalent results.

Dissimilarity ratings were normalized within subjects (by dividing each by the grand mean), then averaged across subjects. The same procedure was followed with the adjective ratings (see Tables 2 and 3). In order to relate dissimilarity ratings to adjective ratings, the absolute difference in (normalized) ratings assigned to the two stimuli in each pair was computed for each textural continuum. These difference scores denoted the difference between pairs of surfaces on specific perceptual dimensions. Dissimilarity ratings were then regressed onto the difference scores along all four textural continua, in order to assess the degree of association between these two sets of measurements. We wished to ascertain to what extent the dissimilarity of two textures could be explained in terms of differences in roughness, hardness, warmth, or stickiness.

The analysis of the recordings was similar to that in Bensmaïa and Hollins (2003). A Fourier analysis was performed on the recorded vibrations, using the one-dimensional fast Fourier transform algorithm in MATLAB (Version 6.1, The MathWorks). The power spec- 

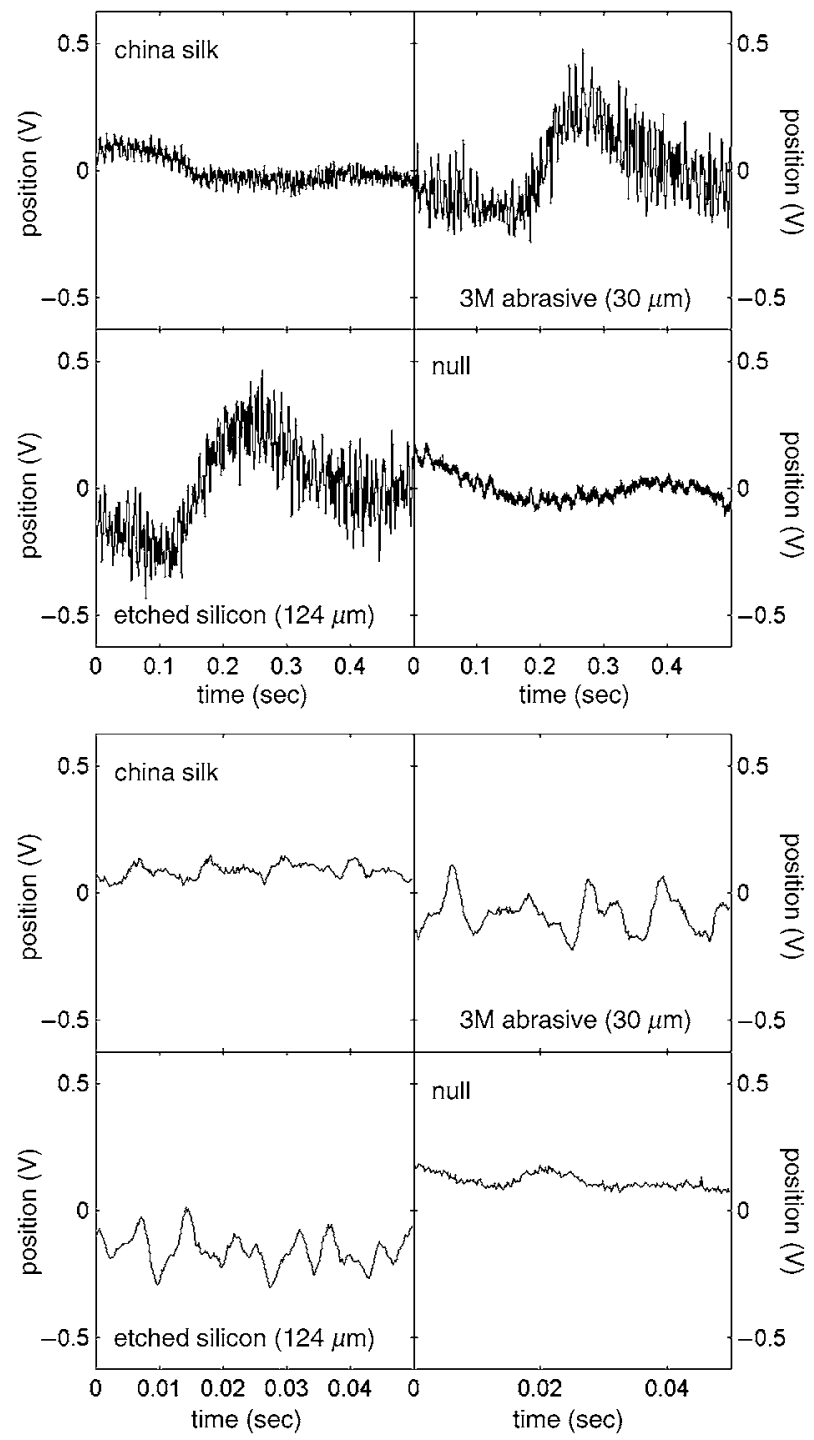

Figure 2. Top panel: raw records obtained from three textured surfaces and the null surface. Bottom panel: raw records obtained from the same three textured surfaces and the null surface shown on an expanded time scale.

trum of each recording for frequencies ranging from 2 to $1500 \mathrm{~Hz}$ was then computed by squaring the complex modulus of each element of its Fourier transform. Then the power spectrum of each null recording was subtracted from the power spectra derived from the two texture recordings obtained on the same sweep.

For each subject, 50 noise-corrected power spectra were obtained for each surface. The median noise-corrected spectrum for each stimulus was then computed for each subject (medians were used, rather than means, to minimize the influence of outliers). If a given median noise-corrected spectral component was negative, it was set to 0 (see Bensmaïa \& Hollins, 2003). The surface spectra obtained from each subject were then normalized by the mean spectral power across surfaces to account for (across-subject) differences in recording gain. Figure 3 shows the noise-corrected power spectra for 8 of the 11 stimuli obtained from 1 of the subjects. The figure reveals obvious differences in the power spectra of vibrations elicited by different surfaces. The spectra that were omitted from this figure re- semble some of those shown. Specifically, the spectra of poplar and paper were indistinguishable from that of birch, and the spectrum of silk charmeuse was virtually identical to that of satin.

Figure 4 shows the noise-corrected power spectra obtained from china silk and etched silicon for all 5 subjects. The similarity of the spectra derived for the same surface from different subjects is striking. The similarity of the spectra across subjects, observed for all the surfaces, justified the pooling of the spectral data.

The primary objective of this study was to ascertain the extent to which the perceived dissimilarity of two surfaces can be explained in terms of differences in the vibrations these surfaces elicit in the skin. In order to relate spectral differences to perceived dissimilarity, we required a measure of the extent to which the neural representations of two texture-induced vibrations differ. Since it has been shown that the Pacinian system mediates the perception of the roughness of fine textures (Bensmaïa \& Hollins, 2003; Hollins et al., 2001), we assumed that it is the transduction and processing of texture-induced vibrations within this sensory channel that are perceptually relevant. Accordingly, we invoked a model describing the information conveyed by the Pacinian system about vibratory waveform. The model has been shown to predict the discriminability of simple and polyharmonic vibrations that vary in intensity and frequency content (see Bensmaïa, Hollins, \& Yau, 2005). From the model, the Pacinian representation of the vibrations produced by each textured surface was characterized, and a measure of the difference between pairs of representations was derived. To gauge the degree to which the Pacinian system contributes to the overall percept, we assessed the extent to which perceived surface dissimilarity could be explained in terms of the Pacinian representations of texture-induced vibrations.

First, the power of each component was weighted according to the frequency sensitivity of the Pacinian system (see Bensmaiia \& Hollins, 2003; Bensmaïa et al., 2005; Makous et al., 1995). Specifically, the power of each spectral component was divided by threshold power at its frequency:

$$
P_{\mathrm{S}}(f)=\frac{A_{f}^{2} f^{2}}{T_{f}^{2} f^{2}},
$$

where $P_{\mathrm{S}}(f)$ is the Pacinian-weighted power of the spectral component with frequency $f$ and amplitude $A_{f}$ in Stimulus $S$ and $T_{f}$ is the threshold amplitude at $f$. Since the shape of the threshold frequency function of the Pacinian system can be modeled using a Gaussian filter (Makous et al., 1995), values for $T_{f}$ were obtained by fitting the following function to mean thresholds measured in a previous experiment (Bensmaïa et al., 2005):

$$
\log \left(T_{f}\right)=\alpha-\beta e^{-\frac{(f-\delta)^{2}}{2 \sigma^{2}}}
$$

where $\alpha, \beta, \delta$, and $\sigma$ were free parameters.

The Pacinian representation of each vibratory stimulus was modeled as a pattern of activation in a hypothetical population of frequency-tuned minichannels. Within this framework, the subjective intensity of a simple or complex vibratory stimulus is determined by the overall activation it elicits in the minichannels. In a polyharmonic stimulus, the effective intensity of each spectral component - that is, its contribution to the overall subjective intensity of the stimulusis assumed to be additive with that of the other components. In other words, the subjective intensity is determined by the sum of the effective intensities of all the spectral components. Since subjective intensity has been shown empirically to be a power function of stimulus power (Franzén, 1969; Gescheider, 1976; Marks, 1979; Stevens, 1968; Verrillo, 1982; Verrillo, Fraioli, \& Smith, 1969), effective intensity was modeled as a power function of $P_{\mathrm{S}}(f)$. Since the power function exponent relating subjective intensity to spectral power has been shown to vary from frequency to frequency (Franzén, 1969; Stevens, 1968), it was necessary to characterize the 
Table 2

Mean Normalized Dissimilarity Ratings (to the Right of the Diagonal) and $\mathrm{D}_{\mathrm{S}_{1} \mathrm{~S}_{2}}$ (to the Left of the Diagonal)

\begin{tabular}{|c|c|c|c|c|c|c|c|c|c|c|c|}
\hline Material & $\begin{array}{c}3 \mathrm{M} \\
(30 \mu \mathrm{m})\end{array}$ & $\begin{array}{c}3 \mathrm{M} \\
(12 \mu \mathrm{m})\end{array}$ & Birch & $\begin{array}{c}\text { Silk } \\
\text { Charmeuse }\end{array}$ & $\begin{array}{l}\text { China } \\
\text { Silk }\end{array}$ & Paper & Latex & $\begin{array}{l}\text { Silicon } \\
(80 \mu \mathrm{m})\end{array}$ & $\begin{array}{l}\text { Silicon } \\
(124 \mu \mathrm{m})\end{array}$ & Poplar & Satin \\
\hline $3 \mathrm{M}(30 \mu \mathrm{m})$ & - & 0.80 & 1.56 & 1.47 & 1.03 & 1.21 & 1.28 & 1.05 & 0.43 & 1.49 & 1.41 \\
\hline $3 \mathrm{M}(12 \mu \mathrm{m})$ & 0.80 & - & 0.91 & 0.81 & 0.26 & 0.44 & 1.86 & 0.40 & 0.46 & 0.79 & 0.70 \\
\hline Birch & 1.42 & 0.70 & - & 0.45 & 0.74 & 0.51 & 2.40 & 0.72 & 1.26 & 0.36 & 0.40 \\
\hline Silk charmeuse & 1.46 & 0.73 & 0.38 & - & 0.56 & 0.50 & 2.16 & 0.51 & 1.10 & 0.10 & 0.18 \\
\hline China silk & 0.86 & 0.64 & 0.49 & 0.71 & - & 0.23 & 1.98 & 0.31 & 0.64 & 0.53 & 0.43 \\
\hline Paper & 1.11 & 0.69 & 0.12 & 0.35 & 0.25 & - & 2.12 & 0.42 & 0.83 & 0.44 & 0.34 \\
\hline Latex & 1.51 & 1.83 & 2.16 & 2.11 & 1.93 & 2.14 & - & 1.99 & 1.57 & 2.21 & 2.19 \\
\hline Silicon $(80 \mu \mathrm{m})$ & 1.08 & 0.46 & 1.03 & 0.84 & 1.13 & 1.06 & 1.82 & - & 0.64 & 0.51 & 0.43 \\
\hline Silicon $(124 \mu \mathrm{m})$ & 0.92 & 0.84 & 1.20 & 1.21 & 1.21 & 1.15 & 1.52 & 0.49 & - & 1.11 & 1.02 \\
\hline Poplar & 1.31 & 0.77 & 0.07 & 0.32 & 0.34 & 0.08 & 2.06 & 1.11 & 1.15 & - & 0.11 \\
\hline Satin & 1.41 & 0.60 & 0.20 & 0.16 & 0.68 & 0.21 & 2.24 & 1.02 & 1.27 & 0.21 & - \\
\hline
\end{tabular}

value of the exponent for frequencies from 2 to $1500 \mathrm{~Hz}$. To that end, we fit a logarithmic function relating exponents obtained in a previous study (Bensmaïa et al., 2005) to stimulus frequency (see Figure 5). ${ }^{3}$

The activation elicited in a minichannel by a given stimulus was described as the summed effective intensity of all the frequency components of the stimulus, weighted according to the channel's frequency tuning. The tuning curve of each minichannel was described as a Gaussian with central frequency $f_{\mathrm{c}}$ and standard deviation $\alpha f_{\mathrm{c}}$.

Center frequencies were spaced logarithmically from 1 to $1500 \mathrm{~Hz}$. Parameter $\alpha$ was set to the value obtained in Bensmaïa et al. (2005), adjusted to take into account the temporal uncertainty in the power spectra. Given that motor speed varied within $15 \%$ of its nominal value, the measured frequency of the spectral components fluctuated in proportion. Since parameter $\alpha$ was derived using idealized power spectra, it took into account measurement error along the frequency axis; the estimate derived in the previous study tends to underestimate the degree to which frequency information is conveyed by the Pacinian system if temporal uncertainty is present in the data. Through simulations, we found that reducing the standard deviation of the Gaussians from 0.43 (see Bensmaïa et al., 2005) to 0.35 corrected for the temporal uncertainty in the input spectra (note that this adjustment had little effect on model predictions). The activation elicited by Stimulus $S$ in the minichannel with central frequency $f_{\mathrm{c}}$ was given by

$$
Z_{f_{\mathrm{c}}}(\mathrm{S})=\sum_{f}\left[P_{\mathrm{S}}(f)\right]^{a_{f}} \cdot e^{-\frac{\left(f-f_{\mathrm{c}}\right)^{2}}{2\left(\alpha f_{\mathrm{c}}\right)^{2}}} .
$$

Figure 6 shows the patterns of activation elicited in the hypothetical population of minichannels by two textured surfaces and the absolute difference between these.

Table 3

\begin{tabular}{lcccc}
\multicolumn{5}{c}{ Mean Normalized Ratings Along the Four Textural Continua } \\
\hline \multicolumn{1}{c}{ Material } & Roughness & Hardness & Warmth & Stickiness \\
\hline $3 \mathrm{M}(12 \mu \mathrm{m})$ & 0.87 & 0.94 & 1.00 & 0.81 \\
$3 \mathrm{M}(30 \mu \mathrm{m})$ & 1.65 & 1.10 & 1.17 & 1.34 \\
Birch & 0.76 & 0.94 & 0.89 & 0.54 \\
China silk & 1.39 & 1.16 & 1.05 & 0.78 \\
Latex & 1.69 & 0.90 & 1.27 & 3.19 \\
Paper & 0.98 & 1.03 & 0.97 & 0.64 \\
Poplar & 0.67 & 0.90 & 0.90 & 0.58 \\
Satin & 0.47 & 0.75 & 1.00 & 0.44 \\
Silicon $(124 \mu \mathrm{m})$ & 1.45 & 1.14 & 0.97 & 1.36 \\
Silicon $(80 \mu \mathrm{m})$ & 0.70 & 1.19 & 0.90 & 0.94 \\
Silk charmeuse & 0.36 & 0.96 & 0.88 & 0.37 \\
\hline
\end{tabular}

In the model, perceived dissimilarity between two vibrations was expressed as the difference in the pattern of activation that they elicited in the population of minichannels. (We used 100 minichannels, but model fit was highly robust to changes in their number beyond about 20; see Bensmaïa et al., 2005.) Specifically, perceived dissimilarity was given by the absolute difference in the activation elicited in each channel by two stimuli, summed across all channels, divided - following Ekman's (1956) law_by the mean activity elicited by the two stimuli:

$$
D_{\mathrm{S}_{1} \mathrm{~S}_{2}}=\frac{\sum_{f_{\mathrm{c}}}\left|Z_{\mathrm{S}_{1}}\left(f_{\mathrm{c}}\right)-Z_{\mathrm{S}_{2}}\left(f_{\mathrm{c}}\right)\right|}{\frac{1}{2} \sum_{f_{\mathrm{c}}}\left[Z_{\mathrm{S}_{1}}\left(f_{\mathrm{c}}\right)+Z_{\mathrm{S}_{2}}\left(f_{\mathrm{c}}\right)\right]} .
$$

In the context of the present experiment, $D_{\mathrm{S}_{1} \mathrm{~S}_{2}}$ is a measure of the difference between the (Pacinian) representation of the vibrations elicited by surface $S_{1}$ and the representation of the vibrations elicited by surface $S_{2}$. This measure of spectral dissimilarity has been found to be a reliable predictor of subjects' tendency to judge pairs of simple and/or complex vibrotactile stimuli as different (Bensmaïa et al., 2005).

We wished to assess the extent to which the spectral dissimilarity of two texture-induced vibrations could account for the overall perceived dissimilarity of the surfaces that produced them. To that end, dissimilarity ratings were regressed on $D_{\mathrm{S}_{1} \mathrm{~S}_{2}}$. Then, in order to determine whether the variance in the dissimilarity ratings not accounted for by spectral differences was associated with any or all of the textural continua, the residuals of the regression relating dissimilarity ratings to $D_{\mathrm{S}_{1} \mathrm{~S}_{2}}$ were regressed on the adjectival difference scores along each of the four continua. Since we had found in a previous experiment that the perception of roughness for fine textures is a function of the Pacinian-weighted power of texture-elicited vibrations (Bensmaïa \& Hollins, 2003), we expected the contribution of differences in roughness to perceived dissimilarity to be subsumed by $D_{\mathrm{S}_{1} \mathrm{~S}_{2}}$.

We also wished to determine the extent to which intensity information alone can account for the perceived differences between surfaces. To that end, we computed the difference in intensity of the two stimuli in each pair. Our intensive difference measure-denoted $D_{\mathrm{S}_{1} \mathrm{~S}_{2}}^{*}$ - gauges the degree to which two stimuli differ in subjective intensity, while ignoring differences in frequency content. The perceived intensity of a stimulus was given by

$$
I_{\mathrm{S}}=\sum_{f} P_{\mathrm{S}}(f)^{a_{f}}
$$

For $a_{f}$, we used values inter- and extrapolated from Franzén's (1969) measurements (see above). $I_{\mathrm{S}}$ is analogous to the measure of Pacinian-weighted power employed by Makous et al. (1995) and Bensmaïa and Hollins (2003), except that it does not assume that 


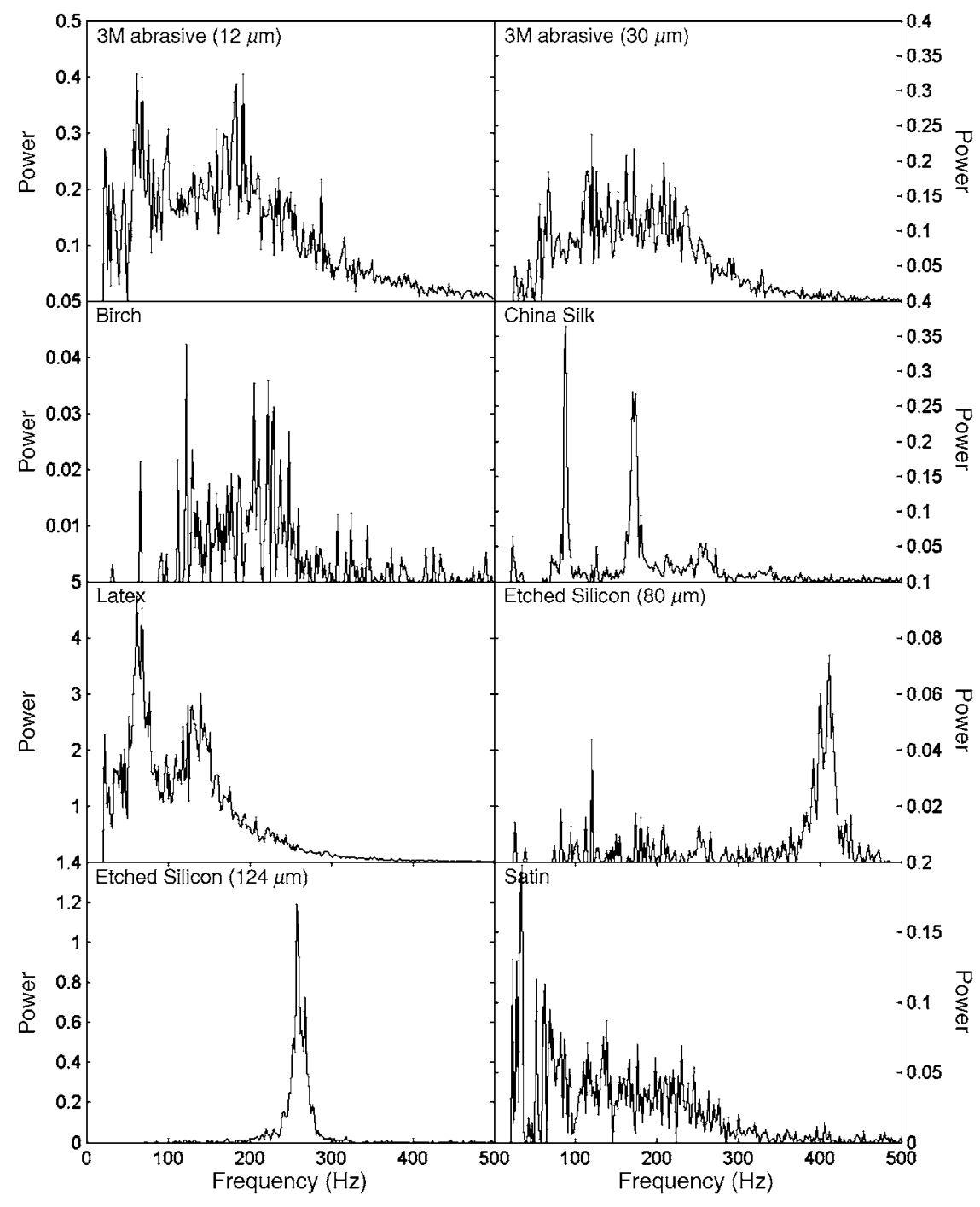

Figure 3. Median noise-corrected spectra of the vibrations produced by 8 of the 11 textured surfaces, obtained from 1 subject. The spectra of poplar and paper are visually indistinguishable from that of birch. The spectrum of silk charmeuse is virtually identical to that of satin.

the exponent relating effective to objective intensity is the same across frequencies (cf. the intensive model in Bensmaïa et al., 2005). The difference in subjective intensity of the vibrations produced by $S_{1}$ and $S_{2}, D_{\mathrm{S}_{1} \mathrm{~S}_{2}}^{*}$, was given by

$$
D_{\mathrm{S}_{1} \mathrm{~S}_{2}}^{*}=\frac{\left|I_{\mathrm{S}_{1}}-I_{\mathrm{S}_{2}}\right|}{\frac{1}{2}\left(I_{\mathrm{S}_{1}}+I_{\mathrm{S}_{2}}\right)} \text {. }
$$

We compared the extent to which variance in the dissimilarity ratings was accounted for by $D_{\mathrm{S}_{1} \mathrm{~S}_{2}}$ and by $D_{\mathrm{S}_{1} \mathrm{~S}_{2}}^{*}$ by regressing the dissimilarity ratings on these two spectral dissimilarity measures.

\section{RESULTS}

\section{Relating Perceived Dissimilarity to Adjectival Difference Scores}

The mean normalized dissimilarity ratings are tabulated in Table 2, and the mean normalized adjective rat- ings are tabulated in Table 3. In order to determine the extent to which perceived dissimilarity was a function of differences along the four textural continua, we performed a multiple regression, with dissimilarity ratings as the dependent variable and difference scores along the four continua as the independent variables. Differences in roughness and stickiness were found to be highly significant predictors of perceived dissimilarity $(\beta=.179$ and $0.764 ; t=2.85$ and 10.46 , respectively, $p<.01$ for both); differences in warmth were marginally significant $(\beta=.123 ; t=1.784, p=.08)$; differences in hardness were nonsignificant $(\beta=-.015 ; t=-0.227, p>.5)$. The fact that hardness was not a significant predictor of dissimilarity is not surprising, given that subjects were instructed to exert the same amount of pressure on the surface throughout the sweep. Hardness cues were minimized as a consequence. Furthermore, the surfaces were 


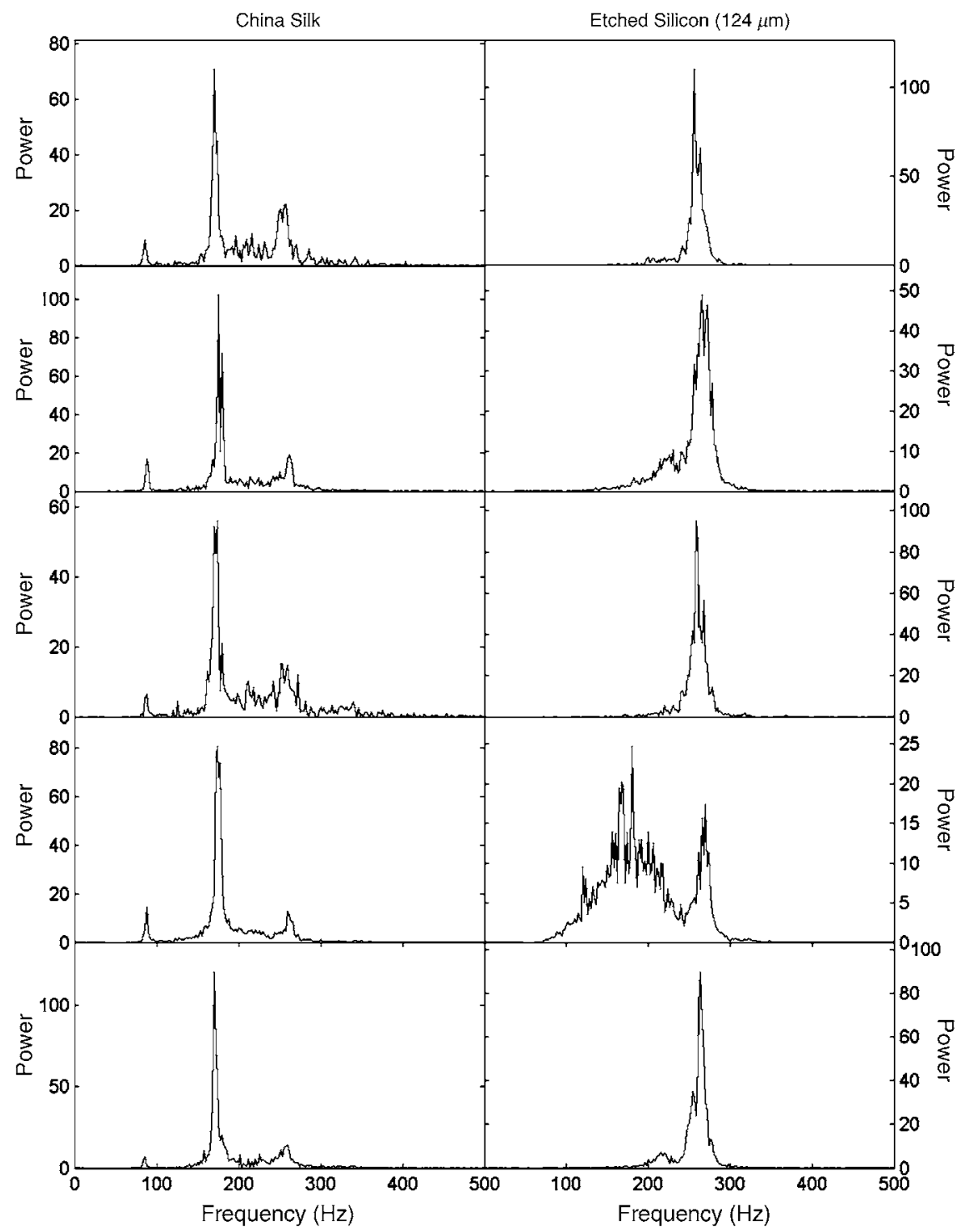

Figure 4. Normalized, median noise-corrected power spectra of vibrations elicited by china silk and etched silicon ( (spatial period $=124 \mu \mathrm{m}$ ), obtained from the 5 subjects. Note the similarity of the spectra obtained from the same surface.

mounted on Plexiglas, which may have homogenized them in objective hardness as well. Most of the variance in the dissimilarity ratings $(86.4 \%)$ could be accounted for by differences along the four textural continua.

\section{Relating Perceived Dissimilarity to Spectral Dissimilarity}

Figure 7 shows the relationship between the dissimilarity ratings and spectral dissimilarity, multiplicatively scaled for maximum overlap. (The mean ratings and corresponding $D_{\mathrm{S}_{1} \mathrm{~S}_{2}}$ are shown in Table 3 ). $D_{\mathrm{S}_{1} \mathrm{~S}_{2}}$ accounted for $81.6 \%$ of the variance in the dissimilarity ratings $(t=$ $14.7, p<.0001)$. In other words, the perceived dissimilarity of a pair of textures could be explained almost en- tirely in terms of differences in the vibrations that they produced in the skin. This is particularly striking given that the model parameters ( $\alpha$ and $a_{f}$ ) were derived from data obtained in other experimental contexts.

$D_{\mathrm{S}_{1} \mathrm{~S}_{2}}^{*}$ accounted for $74.9 \%$ of the variance in the dissimilarity ratings $(t=14.1, p<.0001)$. Both models thus accounted for a large proportion of the variance in the dissimilarity data; however, the increment in $R^{2}$ produced by including nonintensive information was significant $\left(\Delta R^{2}=.067 ; F=19.1, p<.001\right)$. In other words, differences in the perceived intensity of texture-induced vibrations were principally responsible for the perceived dissimilarity of the textures, but temporal factors also played a significant role. 


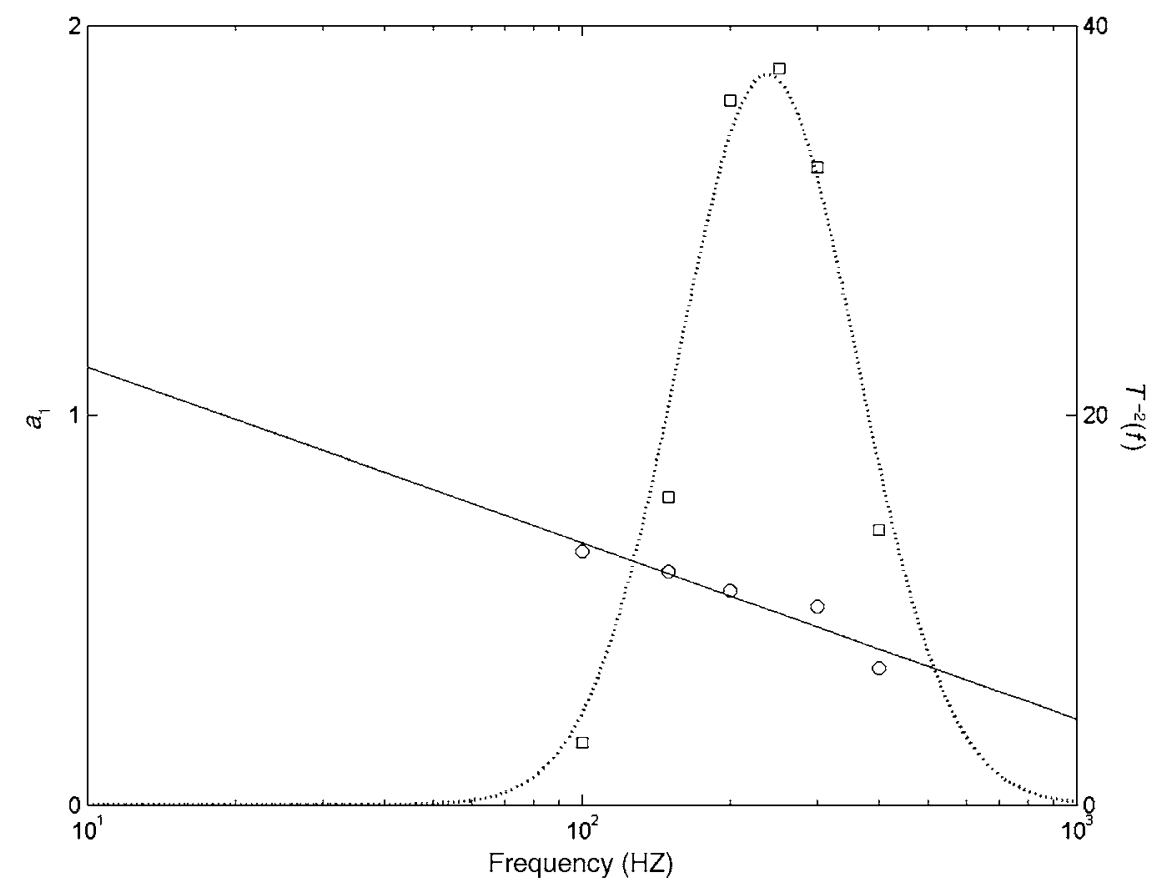

Figure 5. Exponents relating subjective intensity to stimulus power (from Bensmaïa, Hollins, \& Yau, 2005) as a function of frequency (open circles) and the logarithmic function fit to these values (solid line). The dotted line denotes the Pacinian filter (the inverse of the squared threshold) fit to thresholds obtained in Bensmaïa et al. (2005). The inverse square of the measured thresholds is also shown (open squares).

When the residuals of the regression relating perceived dissimilarity to $D_{\mathrm{S}_{1} \mathrm{~S}_{2}}$ were regressed on the adjectival difference scores, only the relationship between the residuals and the warmth difference scores was found to be significant $(t=-0.09,2.8,0.03$, and 0.02 for roughness, warmth, hardness, and stickiness, respectively; $p<.01$ for warmth). Note that differences in hardness were not reliable predictors of perceived dissimilarity even before the variance accounted for by $D_{\mathrm{S}_{1} \mathrm{~S}_{2}}$ was partialed out.

\section{DISCUSSION}

\section{Pacinian-Mediated Texture Perception}

The central result of the present study, then, was that differences in the Pacinian representations of textureinduced vibrations accounted for the bulk of the variance in overall perceived dissimilarity: $D_{\mathrm{S}_{1} \mathrm{~S}_{2}}$ accounted for nearly $82 \%$ of the variance in the dissimilarity ratings. In other words, textures were perceived as dissimilar insofar as the vibrations they produced in the skin were dissimilar. Given that the model parameters were fixed according to previously reported frequency and amplitude response characteristics of the Pacinian system (Bensmaïa et al., 2005), the results are consistent with the idea that this sensory channel plays a key role and conclusively demonstrate that it is capable of conveying suffi- cient information to mediate the identification and discrimination of fine textures.

Furthermore, only warmth difference scores were related to the remaining variance, which (1) suggests that the Pacinian-mediated representation of texture-induced vibrations comprises information about roughness and stickiness and (2) confirms that the perception of thermal conductivity does not rely on vibratory cues (at least, not exclusively). On the one hand, these findings are consistent with previous results demonstrating that roughness perception for fine textures is mediated by the Pacinian system (Bensmaïa \& Hollins, 2003; Hollins et al., 2001). On the other hand, our findings suggest that more than just roughness information is conveyed by the vibrotactile system. Indeed, the perception of stickiness also appears to rely, at least in part, on vibratory cues. As the finger skitters across a sticky surface or squeaks on a slippery one, characteristic vibrations are produced in the skin, which may convey information about friction between skin and surface. It has been shown that a person holding an object in a precision grip automatically adjusts grip force to minimize vibration-producing slips of the object (Johansson \& Westling, 1984, 1987); our data indicate that vibrations of this kind may also affect perception of the object's surface. However, the present results permit only tentative conclusions in this regard, since we used only a small set of surfaces and stickiness covaried with other textural properties. 


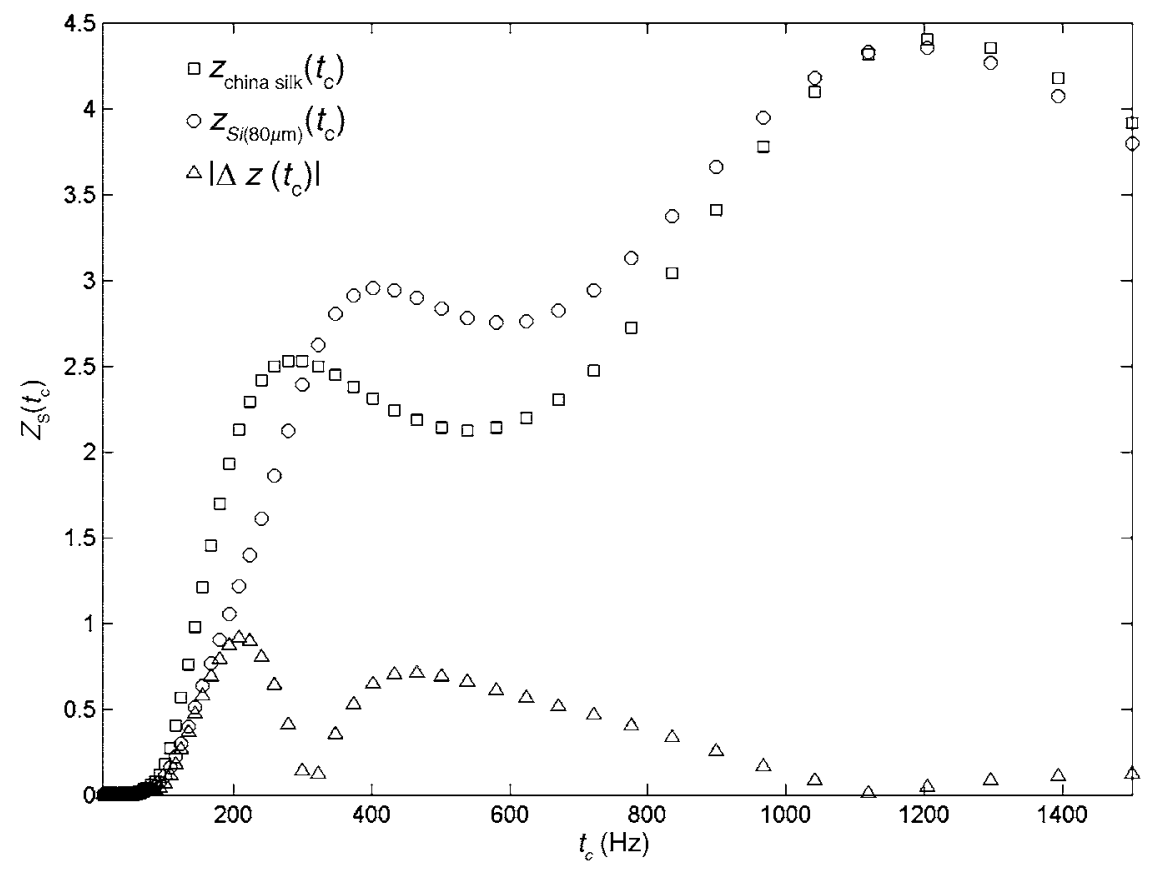

Figure 6. Pattern of activation elicited in the hypothetical population of minichannels by china silk (squares) and that elicited by the etched silicon surface with $80-\mu \mathrm{m}$ spatial period (circles). The triangles denote the absolute difference between these.

\section{Textural Timbre}

A measure describing differences in texture-induced vibrations simply in terms of differences in intensity was found to be a less complete predictor of perceived dissimilarity than was a measure that took into account both temporal and intensive differences. The underlying model describing the Pacinian representation of a vibratory

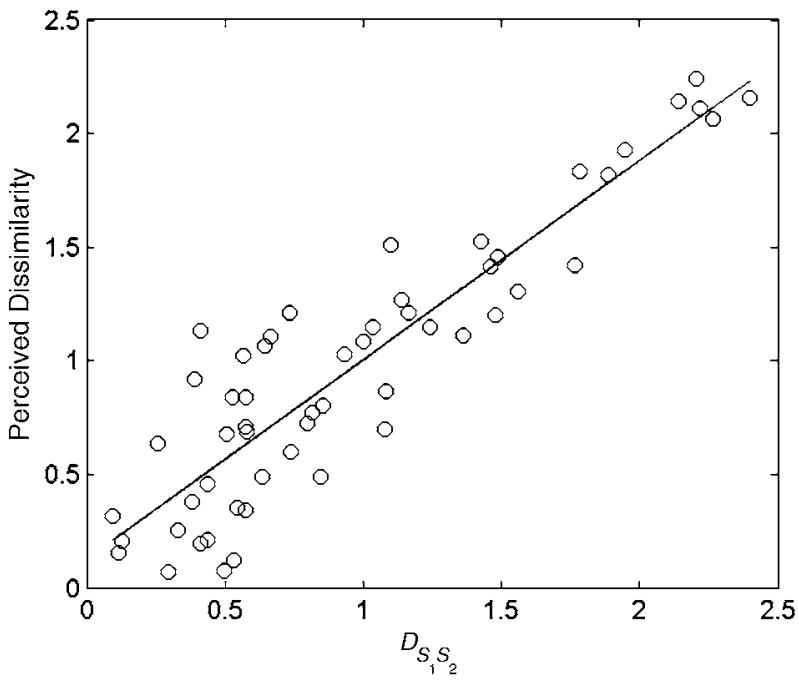

Figure 7. The relationship between perceived dissimilarity and $D_{\mathrm{S}_{1} \mathrm{~S}_{2}}$ is approximately linear. $D_{\mathrm{S}_{1} \mathrm{~S}_{2}}$ accounts for $81.6 \%$ of the variance in perceived dissimilarity. stimulus posits that the Pacinian system conveys both temporal and intensive information. In a previous study (Bensmaïa et al., 2005), both temporal and intensive characteristics of a vibratory stimulus were shown to contribute to perceived differences between vibrations. Hence, the frequency content of texture-elicited vibrations should contribute to perceived differences between surfaces as well. In other words, two surfaces that produce vibrations that are perceptibly different in frequency content should be perceived as different. That $D_{\mathrm{S}_{1} \mathrm{~S}_{2}}$ was a better predictor of perceived dissimilarity than was $D_{\mathrm{S}_{1} \mathrm{~S}_{2}}^{*}$ supports this hypothesis. It is unlikely that temporal information contributes to the dimensional properties of surface percepts - namely, roughness and stickiness (not to mention hardness and warmth). On the one hand, we have found that the temporal features of textureinduced vibrations are not needed to account for surface roughness (Bensmaïa \& Hollins, 2003). Although $D_{\mathrm{S}_{1} \mathrm{~S}_{2}}$ accounts for significantly more variance in roughness ratings than does $D_{\mathrm{S}_{1} \mathrm{~S}_{2}}^{*}\left(\Delta R^{2}=.292 ; F=29.3, p<.01\right)$, the roughest surfaces in the stimulus set happen to have salient temporal signatures (see the spectra for latex, china silk, and $124-\mu \mathrm{m}$ silicon in Figure 3), so differences in roughness are correlated with differences in frequency content. Stickiness is also unlikely to rely on temporal cues, as suggested by the fact that $D_{\mathrm{S}_{1} \mathrm{~S}_{2}}$ does not account for more variance in stickiness ratings than does $D_{\mathrm{S}_{1} \mathrm{~S}_{2}}^{*}\left(\Delta R^{2}=.001 ; F=0.27, p>.5\right)$. In other words, a model comprising both temporal and intensive information does not account for significantly more variance in 
stickiness ratings than does one positing an exclusively intensive representation.

Furthermore, the model posits that frequency content is represented nondimensionally by the Pacinian system: Temporal information about multiple spectral components can be conveyed simultaneously and independently. It is unclear how such a nondimensional perceptual property could result in dimensional features, such as roughness or stickiness. Our findings suggest, then, that fine textures may vary with respect to a previously unidentified perceptual property of textures, which we dub here textural timbre. Textural timbre may be related to what Katz (1925/1989) called the Spezifikationen (identifying characteristics) of fine surfaces - their unique "feel"which, he argued, is more than a particular conjunction of dimensional values.

A note of caution is in order. In our experimental paradigm, textures were scanned passively across the finger at a constant speed. Under the more naturalistic conditions of active touch, however, velocity is not constant. A question remains, then, as to how textural timbre might be affected by changes in scanning velocity. Changing scanning velocity has been shown to have predictable effects on surface roughness (Bensmaïa \& Hollins, 2003). Further research will be required to ascertain whether the proposed model predicts the effects of scanning velocity on timbre or, indeed, on the overall textural percept.

\section{CONCLUSIONS}

In a previous study (Bensmaïa \& Hollins, 2003), we showed that the Pacinian-weighted power of vibrations elicited in the skin by a series of surfaces of the same material, but differing in the scale of their features, predicted their roughness with considerable accuracy. In the present study, we extended this finding by showing that overall perceived dissimilarity between members of a diverse set of fine surfaces - differing in material, as well as in geometrical properties - was well predicted by differences in the vibrations they produced. Differences in roughness, and perhaps in stickiness as well, depend on differences in vibratory power for the contributions that they make to perceived dissimilarity. But more than the power of the vibrations is involved: Purely temporal information is also needed to fully account for the dissimilarity of surfaces. Temporal characteristics of texture-induced vibrations may manifest themselves perceptually as a nondimensional quality, which we dub here textural timbre.

\section{REFERENCES}

Bensmaïa, S. J., \& Hollins, M. (2003). The vibrations of texture. Somatosensory \& Motor Research, 20, 33-43.

Bensmaïa, S. J., Hollins, M., \& Yau, J. (2005). Vibrotactile intensity and frequency information in the Pacinian system: A psychophysical model. Perception \& Psychophysics, 67, 828-841.
Blake, D. T., Hsiao, S. S., \& Johnson, K. O. (1997). Neural coding mechanisms in tactile pattern recognition: The relative contributions of slowly and rapidly adapting mechanoreceptors to perceived roughness. Journal of Neuroscience, 17, 7480-7489.

Connor, C. E., Hsiao, S. S., Phillips, J. R., \& Johnson, K. O. (1990). Tactile roughness: Neural codes that account for psychophysical magnitude estimates. Journal of Neuroscience, 10, 3823-3836.

Connor, C. E., \& Johnson, K. O. (1992). Neural coding of tactile texture: Comparisons of spatial and temporal mechanisms for roughness perception. Journal of Neuroscience, 12, 3414-3426.

EKMAN, G. (1956). Discriminal sensitivity on the subjective continuum. Acta Psychologica, 12, 233-243.

FranZÉN, O. (1969). The dependence of vibrotactile threshold and magnitude functions on stimulation frequency and signal level: A perceptual and neural comparison. Scandinavian Journal of Psychology, 10, 289-298.

GeSCHEIDER, G. A. (1976). Evidence in support of the duplex theory of mechanoreception. Sensory Processes, 1, 68-76.

Hollins, M., Bensmaïa, S. J., Karlof, K., \& Young, F. (2000). Individual differences in perceptual space for tactile textures: Evidence from multidimensional scaling. Perception \& Psychophysics, 62, 1534-1544.

Hollins, M., BensmaÏA, S. J., \& Risner, S. R. (1998). The duplex theory of tactile texture perception. In S. Grondin \& Y. Lacouture (Eds.), Fechner Day 98: Proceedings of the Fourteenth Annual Meeting of the International Society for Psychophysics (pp. 115-120). Quebec: International Society for Psychophysics.

Hollins, M., Bensmaïa, S. J., \& Washburn, S. (2001). Vibrotactile adaptation impairs discrimination of fine, but not coarse, textures. Somatosensory \& Motor Research, 18, 253-262.

Hollins, M., Fox, A., \& Bishop, C. (2000). Imposed vibration influences perceived tactile smoothness. Perception, 29, 1455-1465.

Hollins, M., \& Risner, S. R. (2000). Evidence for the duplex theory of tactile texture perception. Perception \& Psychophysics, 62, 695705 .

Johansson, R. S., \& Westling, G. (1984). Roles of glabrous skin receptors and sensorimotor memory in automatic control of precision grip when lifting rougher or more slippery objects. Experimental Brain Research, 56, 550-564.

Johansson, R. S., \& Westling, G. (1987). Signals in tactile afferents from the fingers eliciting adaptive motor responses during precision grip. Experimental Brain Research, 66, 141-154.

Katz, D. (1989). The world of touch (L. E. Krueger, Trans.). Hillsdale, NJ: Erlbaum. (Original work published 1925)

Lederman, S. J., Loomis, J. M., \& Williams, D. A. (1982). The role of vibration in the tactual perception of roughness. Perception \& Psychophysics, 32, 109-116.

Lederman, S. J., \& TaYlor, M. M. (1972). Fingertip force, surface geometry, and the perception of roughness by active touch. Perception \& Psychophysics, 12, 401-408.

Makous, J. C., Friedman, R. M., \& Vierck, C. J. (1995). A critical band filter in touch. Journal of Neuroscience, 15, 2808-2818.

MARKS, L. E. (1979). Summation of vibrotactile intensity: An analog to auditory critical bands? Sensory Processes, 3, 188-203.

Stevens, S. S. (1968). Tactile vibration: Change of exponent with frequency. Perception \& Psychophysics, 3, 223-228.

TAYLOR, M. M., \& LEDERMAN, S. J. (1975). Tactile roughness of grooved surfaces: A model and the effect of friction. Perception \& Psychophysics, 17, 23-36.

VERRILlo, R. T. (1982). Effects of aging on the suprathreshold responses to vibration. Perception \& Psychophysics, 32, 61-68.

Verrillo, R. T., Fraioli, A. J., \& Smith, R. L. (1969). Sensation magnitude of vibrotactile stimuli. Perception \& Psychophysics, 6, 366372.

Yoshioka, T., Gibb, B., Dorsch, A. K., Hsiao, S. S., \& Johnson, K. O. (2001). Neural coding mechanisms underlying perceived roughness of finely textured surfaces. Journal of Neuroscience, 21, 6905-6916. 


\section{NOTES}

1. Data obtained from S.B. were indistinguishable from those obtained from the other subjects.

2. Regarding the latter criterion, we found that the signal produced by an etched silicon surface with spatial period of $16 \square \mu \mathrm{m}$ (particle size $=$ $4 \square \mu \mathrm{m}$ ) could not be reliably resolved with the apparatus used in our previous study (Bensmaïa \& Hollins, 2003). Although the apparatus used in the present study yielded greater sensitivity, we avoided surfaces with particle sizes less than about $10 \square \mu \mathrm{m}$ as a precaution.

3. To test the parameter dependence of the findings, we carried out the analyses described below, fitting a logarithmic function to exponents obtained by Franzén (1969), and obtained similar results.

(Manuscript received September 15, 2003; revision accepted for publication August 30, 2004.) 